\title{
Robust Nonlinear Optimal Control of Dynamic Systems with Affine Uncertainties
}

\author{
Boris Houska ${ }^{1}$
}

\begin{abstract}
In this paper we present novel strategies to formulate and solve nonlinear robust optimal control problems for dynamic systems which are affine in the uncertainty. We suggest the definition of a constrained Lyapunov differential equation providing robustness interpretations with respect to $L_{2}$-bounded disturbances in the context of inequality state constraints. This interpretation allows us to compute the robust counterpart formulation for optimal control problems which are affine in the uncertainty. Furthermore, we demonstrate the applicability of the presented formulation for a numerical test example: a crane should carry a mass from one to another point while an unknown force excites the open-loop controlled system. The robustly optimized input allows us to control the mass to a target region while satisfying inequality constraints on the worst-case excitation.
\end{abstract}

Key words: Robust Optimization, Optimal Control Problems, Lyapunov Differential Equations

\section{INTRODUCTION}

Since decades robust optimal control problems have received much attention, and robust optimal control for linear systems is a well-developed field [17], [3], [4]. When nonlinear systems are concerned much less approaches exist. However, in [15] or in [8] techniques are proposed that optimize the robustness of nonlinear systems in a linear approximation.

In this paper we are interested in the robust optimization of open-loop controlled uncertain systems that are linear in the uncertain states and disturbances but possibly nonlinear in the remaining states and the control input. Note that this class of problems is slightly different from the class of linear parameter varying systems that are often considered in the literature [2], [6], [13], [12], [16] as in our formulation the uncertainties enters linearly while the control input enters in a nonlinear way. The main challenge is to robustly regard inequality state constraints. For this aim we start in Section II by introducing Lyapunov differential equations as a wellknown tool [14], [7], [10], [11], [5] to compute variancecovariance matrix functions.

In Section III we suggest a definition of constrained Lyapunov differential equations for linear systems with linear inequality constraints providing robustness interpretations for stochastic as well as for $L_{2}$-bounded disturbances. We

\footnotetext{
${ }^{1}$ Optimization in Engineering Center (OPTEC), ESAT-SCD, K. U. Leuven, Kasteelpark Arenberg 10, B-3001 LeuvenHeverlee, Belgium. (boris.houska@esat.kuleuven.be), (moritz.diehl@esat.kuleuven.be)
}

Moritz Diehl ${ }^{1}$

transfer these results in Section IV to special optimal control problems that are linear in the uncertain states and uncertainties while the remaining states and the control input enters in a possibly nonlinear way.

Finally, we apply our method in Section V and Section VI to a crane model where we first optimize the control inputs of a crane in such a way that it carries a mass from one to another point in minimum time. And second, we compare this optimal solution with the case that we take additional model uncertainties, namely a random force acting on the mass, into account.

\section{UnCERTAIN LINEAR TIME-VARYING SyStEMS}

Let us consider a linear time-varying system with a differential state vector $x: \mathbb{R} \rightarrow \mathbb{R}^{n_{x}}$ that is excited by a disturbance function $w: \mathbb{R} \rightarrow \mathbb{R}^{n_{w}}$ :

$$
\begin{aligned}
\dot{x}(t) & =A(t) x(t)+B(t) w(t) \\
y(t) & =C(t) x(t) \\
x(0) & =B_{0} w_{0}
\end{aligned}
$$

with $t \in \mathbb{T}$ and $\mathbb{T}:=[0, T] \subset \mathbb{R}$, where $T$ is the length of the time horizon. Here, we assume that the coefficients $A$ : $\mathbb{T} \rightarrow \mathbb{R}^{n_{x} \times n_{x}}, B: \mathbb{T} \rightarrow \mathbb{R}^{n_{x} \times n_{w}}$, and $C: \mathbb{T} \rightarrow \mathbb{R}^{n_{y} \times n_{x}}$ are squareintegrable functions on the finite interval $\mathbb{T}$. In addition, $y: \mathbb{T} \rightarrow \mathbb{R}^{n_{y}}$ is called the output function while $w_{0} \in \mathbb{R}^{n_{w_{0}}}$ is an uncertainty affecting the initial state via the matrix $B_{0} \in \mathbb{R}^{n_{x} \times n_{w_{0}}}$. At this point in the paper, it is worth to mention that we will later also regard a time dependent function $v$ to be optimized which might enter $A, B$, and $C$ in a possibly nonlinear way, but for the moment we suppress this dependence on $v$ to achieve a convenient notation.

The fundamental solution $G: \mathbb{T} \times \mathbb{T} \rightarrow \mathbb{R}^{n_{x} \times n_{x}}$ of (1) is the unique solution of the initial value problem:

$$
\frac{\partial G(t, \tau)}{\partial t}=A(t) G(t, \tau) \quad \text { with } \quad G(\tau, \tau)=\mathbb{I}
$$

for all $t, \tau \in \mathbb{T}$. Using this notation, we can write the output function $y$ in the form [17]

$$
y(t)=H_{t}^{0} w_{0}+\int_{0}^{T} H_{t}(\tau) w(\tau) \mathrm{d} \tau
$$

with the Green's or impulse response function $H_{t}: \mathbb{T} \rightarrow$ $\mathbb{R}^{n_{y} \times n_{w}}$ being defined by

$$
\forall t, \tau \in \mathbb{T}: H^{t}(\tau):= \begin{cases}C(t) G(t, \tau) B(\tau) & \text { if } \tau \leq t \\ 0 & \text { otherwise }\end{cases}
$$


and $H_{t}^{0}:=C(t) G(t, 0) B_{0}$. Obviously, the differential equation (2) for the fundamental solution $G$ is completely independent of the matrix functions $B$ and $C$. In order to describe the dependence of the state on the disturbance $\omega:=\left(w_{0}, w(\cdot)\right)$, we make use of Lyapunov differential equations of the form

$$
\begin{aligned}
\dot{P}(t) & =A(t) P(t)+P(t) A(t)^{T}+B(t) B(t)^{T} \\
P(0) & =B_{0} B_{0}^{T}
\end{aligned}
$$

The state of this differential equation is a matrix valued function $P: \mathbb{T} \rightarrow \mathbb{R}^{n_{x} \times n_{x}}$. Note that $P$ propagates the following important information [10], [11], [5]: if the disturbance $w$ entering the system (1) is a Gaussian white noise process with

$$
\begin{aligned}
& \mathbb{E}\{w(t)\}=0 \quad \text { and } \\
& \mathbb{E}\left\{w_{i}\left(t_{1}\right) w_{j}\left(t_{2}\right)^{T}\right\}=\Sigma\left(t_{1}\right) \delta\left(t_{2}-t_{1}\right) \delta_{i, j}
\end{aligned}
$$

for all $t, t_{1}, t_{2} \in \mathbb{T}$ and for all $i, j \in\left\{1, . ., n_{w}\right\}$ while the uncertain value $w_{0}$ is Gaussian distributed with variancecovariance matrix $\mathbb{I} \in \mathbb{R}^{n_{x} \times n_{x}}$ then $P(t)$ is the variancecovariance matrix of the state $x(t)$ for all times $t \in \mathbb{T}$. Consequently, the variance-covariance matrix of the output $y$ is at each time $t \in \mathbb{T}$ given by $C(t) P(t) C(t)^{T}$.

\section{INEQUALITY CONSTRAINED LyAPUNOV DIFFERENTIAL EQUATIONS}

As almost all systems in the real-world are subject to inequality constraints we like to discuss the linear system (1) in combination with output constraints of the form

$$
\forall t \in \mathbb{T}, \forall i \in\left\{1, \ldots, n_{y}\right\}: \quad y_{i}(t) \leq d_{i}(t)
$$

The function $d: \mathbb{T} \rightarrow \mathbb{R}^{n_{y}}$ is assumed to be continuous with strictly positive components. Furthermore, $C_{i}(t)$ denotes the $i$-th row vector of $C(t)$. For all theoretical purposes in this section, we will assume $d_{i}(t)=1$ by rescaling the rows $C_{i}(t)$ for all components $i \in I:=\left\{1, \ldots, n_{y}\right\}$. In this case, all constraints have the simple form $y_{i}(t) \leq 1$.

Now, we suggest to consider a corresponding constrained Lyapunov differential equation for the matrix valued function $P$ defined by

$$
\begin{aligned}
& \dot{P}(t)=A(t) P(t)+P(t) A(t)^{T}+B(t) B(t)^{T} \\
& 0 \geq C_{i}(t) P(t) C_{i}(t)^{T}-1 \\
& P(0)=B_{0} B_{0}^{T} .
\end{aligned}
$$

The aim of this section is to discuss a worst case interpretation of the constrained Lyapunov system (7) based on the assumption that the disturbance $\omega:=\left(w_{0}, w(\cdot)\right)$ is a bounded but unknown function. For this aim, we consider the Hilbert space $L_{2}$ of all square-integrable functions from $\mathbb{T}$ to $\mathbb{R}^{n_{w}}$. Moreover, we define an inner product $\langle\cdot \mid \cdot\rangle_{W}: W \times W \rightarrow \mathbb{R}$ in the space $W:=\mathbb{R}^{n_{w_{0}}} \times L_{2}$ and the corresponding $W$-norm $\|\cdot\|_{W}: W \rightarrow \mathbb{R}$ by

$$
\begin{aligned}
\left\langle\omega_{1} \mid \omega_{2}\right\rangle_{W} & :=w_{0,1}^{T} w_{0,2}+\int_{0}^{T} w_{1}(\tau)^{T} w_{2}(\tau) \mathrm{d} \tau \text { and } \\
\left\|\omega_{1}\right\|_{W} & :=\sqrt{\left\langle\omega_{1}, \omega_{1}\right\rangle_{W}}
\end{aligned}
$$

for all $\omega_{1}, \omega_{2} \in W$ and $\omega_{j}=\left(w_{0, j}, w_{j}(\cdot)\right)$ for $j \in\{1,2\}$.

In the next step, we define the ball $\mathscr{B} \subseteq W$ by

$$
\mathscr{B}:=\left\{\omega \in L_{2} \mid\|\omega\|_{W} \leq 1\right\} .
$$

The worst case interpretation of the constrained Lyapunov differential equation can now be stated as follows:

Theorem 3.1: The constraints of the form $y_{i}(t) \leq 1$ are satisfied for all times $t \in \mathbb{T}$, all components $i \in I$, and all disturbances $\omega \in \mathscr{B}$ if and only if the constrained Lyapunov differential equation (7) admits a feasible solution $P$.

Proof: Let us write equation (3) for each component $i \in I$ in the form

$$
y_{i}(t)=\left\langle\mathscr{H}_{t, i}^{T} \mid \omega\right\rangle_{W}
$$

with $\mathscr{H}_{t, i}:=\left(H_{t, i}^{0}, H_{t, i}\right)$ and apply Cauchy's inequality to find an upper bound on the worst case excitation of $y_{i}(t)$ :

$$
\max _{\omega \in \mathscr{B}} y_{i}(t) \leq\left\|\mathscr{H}_{t, i}\right\|_{W}
$$

for all $t \in \mathbb{R}$ and all $i \in I$. Using the fact that

$$
\frac{\mathscr{H}_{t, i}}{\left\|\mathscr{H}_{t, i}\right\|_{W}} \in \mathscr{B}
$$

we recognize that the inequality in (9) is tight, i.e. it can be replaced by an equality. Moreover, as

$$
P(t):=B_{0} B_{0}^{T}+\int_{0}^{t} G(t, \tau) B(\tau) B(\tau)^{T} G(t, \tau)^{T} \mathrm{~d} \tau
$$

solves the Lyapunov differential equation (5) we find

$$
\left\|\mathscr{H}_{t, i}^{T}\right\|_{W}^{2}=C_{i}(t) P(t) C_{i}(t)^{T}
$$

by using the definition of the function $\mathscr{H}_{t, i}$. Thus, we obtain the following relation for the worst case excitation:

$$
\max _{\omega \in \mathscr{B}} y_{i}(t)=\sqrt{C_{i}(t) P(t) C_{i}(t)^{T}},
$$

which implies the statement of the Lemma.

\section{Remark:}

- Provided that $w$ is a unit-bandwidth white noise process, while $w_{0}$ is a Gaussian distributed random variable with variance-covariance matrix $\mathbb{I}$, we have

$$
\begin{aligned}
& \forall t \in \mathbb{R}, i \in I: \\
& \text { Probability }\left\{y_{i}(t)>1\right\}<\int_{1}^{\infty} \frac{1}{\sqrt{2 \pi}} e^{-\frac{1}{2} z^{2}} \mathrm{~d} z .
\end{aligned}
$$

if and only if the constrained Lyapunov differential equation (7) admits a feasible solution $P$. This stochastic single chance interpretation can directly be seen by making use of the fact that we have $\mathbb{E}\left\{y_{i}(t)^{2}\right\}=$ $C_{i}(t) P(t) C_{i}(t)^{T}$ in this case. 


\section{Robust Optimal CONTROL}

Let us transfer the results from the previous section to uncertain optimal control problems of the form

$$
\begin{array}{rll}
\min _{x(\cdot), v(\cdot), T} & & J[v(\cdot), T] \\
\text { s.t. } & & \\
\forall t \in \mathbb{T}: \quad \dot{x}(t)= & A(v(t)) x(t)+B(v(t)) w(t) \\
& +r(v(t)) \\
\forall t \in \mathbb{T}: \quad 0 \quad \geq & C(v(t)) x(t)-d(v(t)) \\
r(0)= & r_{0}(v(0))+B_{0} w_{0} \\
v \in & \mathbb{V}
\end{array}
$$

Here, $v: \mathbb{R} \rightarrow \mathbb{R}^{n_{v}}$ is the nonlinear behavior of the system. This behavior is assumed to lie in a given set $\mathbb{V} \subset L_{2}$ of measurable functions on the time interval $\mathbb{T}$. In the context of optimal control problems, $\mathbb{V}$ has typically the form

$$
\mathbb{V}=\left\{v: \mathbb{T} \rightarrow \mathbb{R}^{n_{v}} \mid \begin{array}{l}
\forall t \in \mathbb{T}: \\
0=F(t, \dot{v}, v) \\
0=G(v(0), v(T))=0 \\
0 \geq H(v(t))
\end{array}\right\}
$$

Note that this set $\mathbb{V}$ can for example in a trivial case contain a constant parameter which could be formulated as

$$
\mathbb{V}_{\text {example }}=\left\{v: \mathbb{T} \rightarrow \mathbb{R}^{n_{v}}: \dot{v}=0\right\},
$$

or piecewise constant controls, or non-trivial implicit differential algebraic equations etc.. However, $\mathbb{V}$ should not depend on $\omega$ - otherwise, the system is in general not affine in the uncertainty.

In our formulation, the objective functional $J$ is assumed to be independent of $x .{ }^{1}$ The coefficients $A, B, C, d$ are now regarded as functions in $v$. Moreover, we have introduced a continuous reference function $r: \mathbb{R}^{n_{v}} \rightarrow \mathbb{R}^{n_{x}}$, which can, analogous to the nominal initial value $r_{0}: \mathbb{R}^{n_{v}} \rightarrow \mathbb{R}^{n_{x}}$, also depend on the behaviour $v$.

To formulate the robust counterpart formulation of the above optimization problem we follow the classical approach [3], i.e. we consider the optimization problem

$$
\begin{array}{ccc}
\min _{v(\cdot), T} & \\
\text { s.t. } & & \\
\forall t \in \mathbb{T}: & \left.0 \geq y^{\max }(t), T\right] \\
& v \in \mathbb{V}(\cdot), T)-d(v(t))
\end{array}
$$

where $y_{i}^{\max }(t ; v(\cdot), T)$ is for each $t \in \mathbb{T}$ and each $i \in I$ defined

\footnotetext{
${ }^{1}$ Note that an affine dependence of $J$ on $x$ can always be eliminated by an introduction of slack parameters.
}

to be the optimal value of the sub-maximization problem

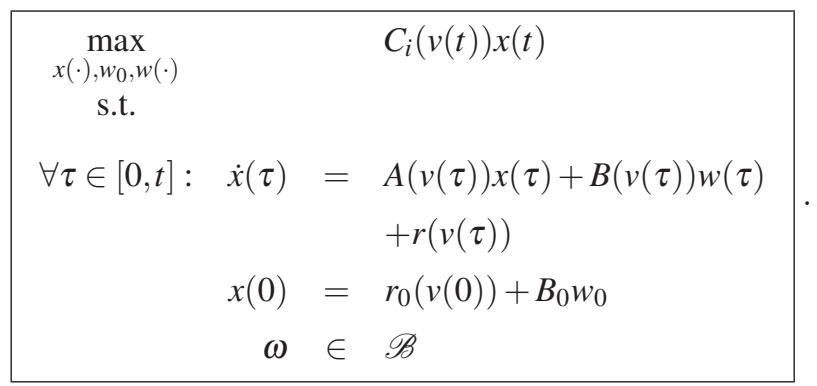

One of the key results of this paper is that the robust counterpart problem (13) can, as a direct consequence of Theorem 3.1, equivalently be written as

$$
\begin{aligned}
& \min _{\substack{x_{r}(\cdot), P(\cdot), v(\cdot), T \\
\text { s.t. }}} J[v(\cdot), T] \\
& \forall t \in \mathbb{T}: \quad \dot{x}_{r}(t)=A(v(t)) x_{r}(t)+r(v(t)) \\
& x_{r}(0)=r_{0}(v(0)) \\
& \forall t \in \mathbb{T}: \quad \dot{P}(t)=A(v(t)) P(t)+P(t) A(v(t))^{T} \\
& +B(v(t)) B(v(t))^{T} \\
& P(0) \quad=B_{0} B_{0}^{T} \\
& \forall i \in I: \quad 0 \quad \geq C_{i}(v(t)) x_{r}(t)-d_{i}(v(t)) \\
& +\sqrt{C_{i}(v(t)) P(t) C_{i}(v(t))^{T}} \\
& v \in \mathbb{V}
\end{aligned}
$$

Note that in this robust counterpart formulation, $x_{r}$ denotes the reference state trajectory, which would be obtained for $\omega=0$.

An interesting point about this robust counterpart formulation is that we regard an infinite dimensional disturbance, represented by $\omega \in W$, while there is also an infinite dimensional number of constraints robustly satisfied as the linear path constraints have to be satisfied for all $t$ in the time interval $\mathbb{T}$. Note that this is a main advantage in comparison to other existing robust counterpart formulations for optimal control problems, which have been proposed in [8], [15].

Finally, we note that optimal control problems which are affine in the uncertainty can often be found in practice. Even if a problem is not in this form, we might be in one of the following situations:

- If we consider a nonlinear dynamic system, the system can at least for small disturbances be robustified in a linear approximation as proposed in [15], [8]. In this case, the reference trajectory is the solution of the nonlinear dynamic system for $\omega=0$, which can be optimized, too. In this case, we do not have many guarantees for larger disturbances but for sufficiently small disturbances the linear approximation is valid.

- If we have a linear system of the form

$$
\dot{x}(t)=\tilde{A}(t) x(t)+B(t) w(t)+D(t) u(t)+r(t)
$$




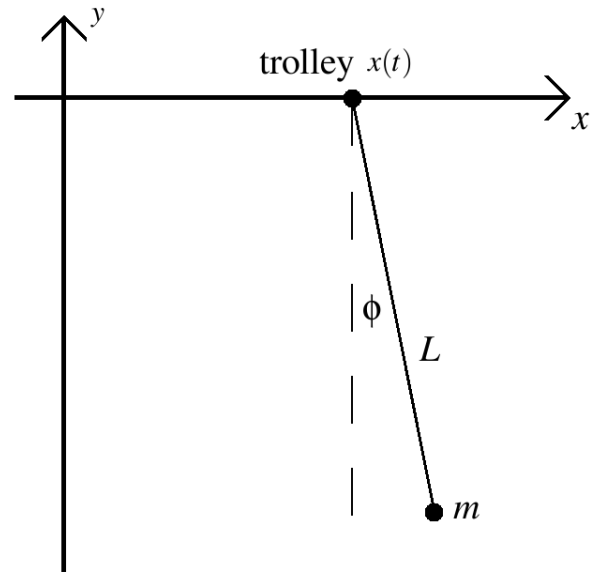

Fig. 1. A scetch of the crane.

with a control function $u$ we might be interested in a linear feedback law of the form $u(t):=u_{\text {ref }}(t)+K(t) x(t)$, where $K(t)$ is a matrix function that should be optimized in such a way that the constraints are robustly satisfied. Now, we can summarize the components of $K$ in a vector valued function $v:=\operatorname{vec}(K)$. If we define

$$
A(v(t)):=\tilde{A}(t)+D(t) K(t)
$$

we can obviously transfer the above robust counterpart formulation. Note that this approach can also take linear control constraints into account. In addition, it can be generalized for the case that a linear estimator gain together with a linear feedback gain should be optimized in a robust way.

\section{Optimal Control of a Crane}

To illustrate the applicability and potential of the robust counterpart formulation (14), we consider a crane, which should carry a mass $m$ from a given point to a given target region (cf. Figure 1): If the crane's cable with length $L$ is very long with respect to the horizontal excitation $L \sin (\phi)$ of the mass, which is affected by an unknown force $F$, it is an easy exercise to show that the dynamics of the angle of the line of the crane can be described by the following differential equation:

$$
\frac{\mathrm{d}}{\mathrm{d} t}\left(\begin{array}{c}
\phi(t) \\
\dot{\phi}(t)
\end{array}\right)=A(t)\left(\begin{array}{c}
\phi(t) \\
\dot{\phi}(t)
\end{array}\right)+B(t) F(t)+r(t)
$$

with

$$
\begin{aligned}
& A(t):=\left(\begin{array}{cc}
0 & 1 \\
-\frac{g}{L(t)} & -\left(b+2 \frac{\dot{L}(t)}{L(t)}\right)
\end{array}\right), \quad B(t):=\left(\begin{array}{c}
0 \\
\frac{1}{m L(t)}
\end{array}\right) \\
& \text { and } \quad r(t):=\left(\begin{array}{c}
0 \\
-\frac{\ddot{x}}{L}-\frac{\dot{L} \dot{x}}{L^{2}}
\end{array}\right)
\end{aligned}
$$

Here, the crane is only considered in a plane $\mathbb{R}^{2}$, in which the mounting point of the cable is at the time $t$ located at the position $(x(t), 0)^{T} \in \mathbb{R}^{2}$ while the mass has the position $(x(t)+L(t) \sin (\phi(t)),-L(t) \cos (\phi(t)))^{T} \in \mathbb{R}^{2}$. In this notation, $g=9.81 \frac{\mathrm{m}}{\mathrm{s}^{2}}$ is the gravitational constant and $b=0.1 \frac{1}{\mathrm{~s}} \mathrm{a}$ friction coefficient. Note that the above model is only valid for small excitations $\phi$ where the dynamics can be linearized in the states $\phi$ and $\dot{\phi}$.

The external force $F$, acting at the mass in horizontal direction, is assumed to be unknown in our example. The optimal control problem we would like to solve now, assumes that we have the control $u:=(\ddot{x}, \ddot{L})$ as a degree of freedom to bring the mass as fast as possible from a given point into a desired target region. More precisely, we define the feasible behaviour

$$
v:=\left(z^{T}, u^{T}\right)^{T}:=\left(x, L, \dot{x}, \dot{L}, u^{T}\right)^{T} \in \mathbb{V}
$$

of the dynamic system by

$\mathbb{V}:=\left\{\begin{array}{l|l}v:[0, T] \rightarrow \mathbb{R}^{n_{6}} & \begin{array}{l}\forall t \in[0, T]: \\ \dot{z}(t)=\left(\dot{x}(t), \dot{L}(t), u(t)^{T}\right)^{T} \\ z(0)=z_{0} \\ z(T)=z_{T} \\ v_{\min } \geq v(t) \geq v_{\max }\end{array}\end{array}\right\}$,

where we use the following values for our example

$$
\begin{aligned}
z_{0} & :=\left(0 \mathrm{~m}, 100 \mathrm{~m}, 0 \frac{\mathrm{m}}{\mathrm{s}}, 0 \frac{\mathrm{m}}{\mathrm{s}}\right)^{T} \\
z_{T} & :=\left(40 \mathrm{~m}, 100 \mathrm{~m}, 0 \frac{\mathrm{m}}{\mathrm{s}}, 0 \frac{\mathrm{m}}{\mathrm{s}}\right)^{T} \\
v_{\min } & :=\left(-10 \mathrm{~m}, 50 \mathrm{~m},-20 \frac{\mathrm{m}}{\mathrm{s}},-20 \frac{\mathrm{m}}{\mathrm{s}},-0.3 \frac{\mathrm{m}}{\mathrm{s}^{2}},-1 \frac{\mathrm{m}}{\mathrm{s}^{2}}\right)^{T} \\
v_{\max } & :=\left(50 \mathrm{~m}, 102 \mathrm{~m}, 20 \frac{\mathrm{m}}{\mathrm{s}}, 20 \frac{\mathrm{m}}{\mathrm{s}}, 0.3 \frac{\mathrm{m}}{\mathrm{s}^{2}}, 1 \frac{\mathrm{m}}{\mathrm{s}^{2}}\right)^{T} .
\end{aligned}
$$

We are first interested in the following minimum time optimal control problem for the case that we have no disturbances, i.e. for $F=0$ :

$$
\begin{array}{rl}
\min _{x(\cdot), v(\cdot), p, T} & T \\
\text { s.t. } & \\
\forall t \in[0, T]: & \frac{\mathrm{d}}{\mathrm{d} t} x(t)=A(v(t)) x(t)+r(v(t)) \\
\forall t \in[0, T]: & \phi_{\min } \leq \phi(t) \leq \phi_{\max } \\
& x(0)=x_{0} \\
& \underline{x}_{\text {target }} \leq x(T) \leq \bar{x}_{\text {target }} \\
& v \in \mathbb{V}
\end{array}
$$



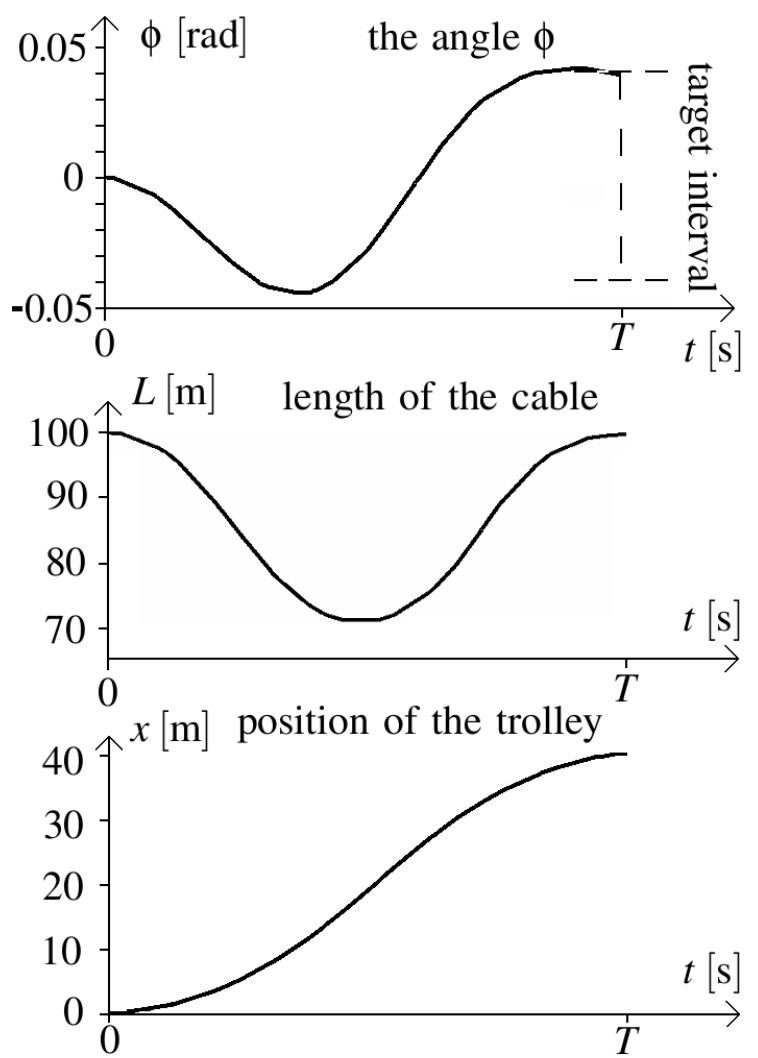

Fig. 2. A locally optimal result of the optimal control problem (16).

where we chose the following numerical values:

$$
\begin{aligned}
x_{0} & :=\left(0 \mathrm{rad}, 0 \frac{\mathrm{rad}}{\mathrm{s}}\right)^{T} \\
\underline{x}_{\text {target }} & :=\left(-0.042 \mathrm{rad},-0.013 \frac{\mathrm{rad}}{\mathrm{s}}\right)^{T} \\
\bar{x}_{\text {target }} & :=\left(0.042 \mathrm{rad}, 0.013 \frac{\mathrm{rad}}{\mathrm{s}}\right)^{T} \\
\phi_{\max } & :=-\phi_{\min }:=0.05 \mathrm{rad} .
\end{aligned}
$$

Now, we use the dynamic optimal control package ACADO [1] to solve the above problem by using a piecewise constant control parametrization in combination with an SQP method. The corresponding locally optimal solution, that was obtained with this method, is shown in Figure 2. Note that the optimal result for the angle $\phi$, the position $x$, and the cable length $L$ are shown. The path constraints for $\phi$ are not active in this optimal solution but note that the target constraint $\phi(T) \leq 0.042 \mathrm{rad}$ is active.

The corresponding value for the minimum time is

$$
T=23.33 \mathrm{~s} .
$$

Note that it is optimal to reduce the cable length during the movement of the crane.
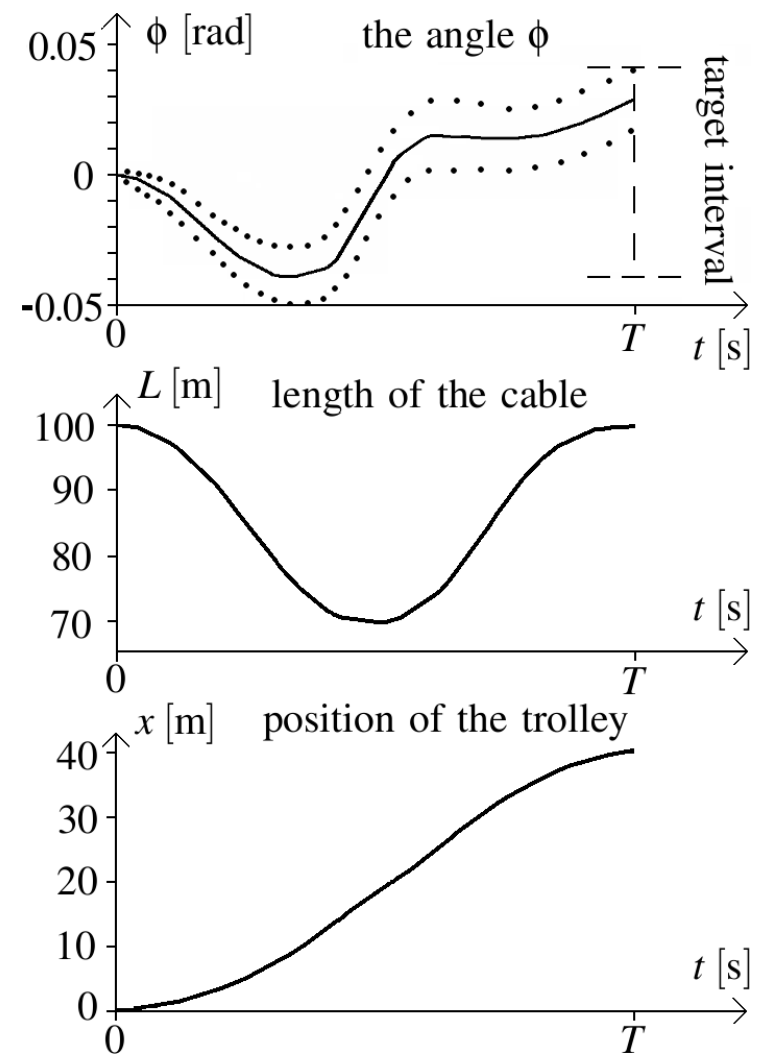

Fig. 3. A locally optimal result for the robust counterpart problem (14) associated with the optimal control problem (16).

\section{Vi. Robust Optimal Control of a Crane}

In this section we discuss the solution of the robust counterpart optimal control problem which is associated with the problem (16). For this aim, we choose $m=600 \mathrm{~kg}$ and assume that the uncertain force $F$ is bounded by

$$
\|F(\cdot)\|_{L_{2}}^{2} \leq 1200 \mathrm{~N}^{2} \mathrm{~s} .
$$

We use the optimization software ACADO again to solve also the robustified optimal control problem taking the Lyapunov equation for the state variances into account. Here, we assume that all states are at the time $t=0$ exactly known, while the path constraints on $\phi$ as well as the target region constraints should be satisfied for all forces $F$ that are bounded by (14). A corresponding robust and locally optimal solution is shown in Figure 3.

The optimal value for the time $T$ is now larger:

$$
T=24.56 \mathrm{~s}
$$

as we need to satisfy more conservative constraints. To visualize that the robustified constraints were active at the optimal solution, which is shown in Figure 3, the functions

$\bar{\phi}:=\phi(t)+\gamma \sqrt{\operatorname{Var}(\phi(t))}$ and $\phi:=\phi(t)-\gamma \sqrt{\operatorname{Var}(\phi(t))}$ 
are plotted as dotted lines. Note that the lower bound of the form

$$
\phi(t)-\sqrt{P_{\phi, \phi}(t)} \geq-0.05 \mathrm{rad}
$$

is at active at a certain time $t \in \mathbb{T}$ while the target constraint

$$
\phi(T)+\sqrt{P_{\phi, \phi}(T)} \leq 0.042 \mathrm{rad}
$$

is also active.

\section{CONCLUSIONS AND OUTLOOK}

We have presented methods to design and optimize the stability and robustness of nonlinear dynamic systems with affine uncertainties taking inequality state constraints into account. After reviewing existing concepts for the robustification of linear systems we concentrated on an extension of Lyapunov differential equations for systems with state constraints. We have summarized the interpretation of such constrained Lyapunov differential equations in Theorem 3.1 together with the associated remarks showing the equivalence between

- the existence of a feasible solution of the constrained Lyapunov differential equation,

- the exact robustness of the underlying constrained linear dynamic system with respect to $L_{2}$-bounded disturbances,

- and the stochastic single chance constraint interpretation for the case of a white noise disturbance.

Furthermore, we transferred the constrained Lyapunov differential equations for special optimal control problems that are linear in the state and the uncertainty while the remaining behaviour can possibly enter in a nonlinear way. In order to demonstrate the applicability of the presented results, we have tested our method for a simple crane model. After a discussion of a time optimal trajectory for this crane we have presented a robustified solution taking inequality state constraints into account.

In the future, we plan to develop numerical methods that use the special structure of Lyapunov differential equations within the nonlinear optimal control algorithms. The method might also be suitable for larger systems than discussed in this paper. Moreover, our approach might be transferred to periodic system, where beside the robustness aspects also the stability of the system can be optimized [9].

\section{Acknowledgements:}

Research supported by Research Council KUL: CoE EF/05/006 Optimization in Engineering(OPTEC), IOF-SCORES4CHEM, GOA/10/009 (MaNet), GOA/10/11, several PhD/postdoc and fellow grants; Flemish Government: FWO: PhD/postdoc grants, projects G.0452.04, G.0499.04, G.0211.05, G.0226.06, G.0321.06, G.0302.07, G.0320.08, G.0558.08, G.0557.08, G.0588.09,G.0377.09, research communities (ICCoS, ANMMM, MLDM); IWT: PhD Grants, Belgian Federal Science Policy Office: IUAP P6/04; EU: ERNSI; FP7-HDMPC, FP7-EMBOCON, Contract Research: AMINAL. Other: Helmholtz-viCERP, COMETACCM.

\section{REFERENCES}

[1] ACADO Toolkit Homepage. www.acadotoolkit.org, 2009.

[2] F. Amato. Robust Control of Linear Systems Subject to Uncertain Time-Varying Parameters. Springer, 2006.

[3] A. Ben-Tal and A. Nemirovski. Robust Convex Optimization. Math. Oper. Res., 23:769-805, 1998.

[4] A. Ben-Tal and A. Nemirovskii. Lectures on Modern Convex Optimization: Analysis, Algorithms, and Engineering Applications. MPSSIAM Series on Optimization. MPS-SIAM, Philadelphia, 2001.

[5] S. Bittanti, P. Bolzern, and P. Colaneri. Stability analysis of linear periodic system via the Lyapunov equation. Proc. 9th IFAC World Congress, Budapest, Hungary, pages 169-172, 1984.

[6] P.A. Bliman. A convex approach to robust stability for linear systems with uncertain scalar parameters. SIAM Journal on Control and Optimization, 42(6):20162042, 2004.

[7] P. Bolzern and P. Colaneri. The periodic Lyapunov equation. SIAM J. Matrix Anal. Appl., 9(4):499-512, 1988.

[8] M. Diehl, H.G. Bock, and E. Kostina. An approximation technique for robust nonlinear optimization. Mathematical Programming, 107:213230, 2006.

[9] B. Houska. Robustness and Stability Optimization of Open-Loop Controlled Power Generating Kites. Master's thesis, University of Heidelberg, 2007.

[10] R.E. Kalman. Control system analysis and design by the second method of Lyapunov. Trans. ASME Ser. D.J. Basic Engrg., 82:pp. 371-400, 1960.

[11] R.E. Kalman. Lyapunov functions for the problem of Lur'e in automatic control. Proc. Nat. Acad. Sci. USA, 49:pp. 201-205, 1963.

[12] M. Kothare, V. Balakrishnan, and M. Morari. Robust constrained model predictive control using linear matrix inequalities. Automatica, 32(10):1361-1379, November 1996.

[13] S. Lim and J.P. How. Analysis of linear parameter-varying systems using a non-smooth dissipative systems framework. International Journal of Robust and Nonlinear Control, DOI: 10.1002/rnc.675:10671092, 2002

[14] M.A. Lyapunov. Probléme general de la stabilité du mouvement. Ann. Fac. Sci. Toulouse Math., 5(9):pp. 203-474, 1907.

[15] Z.K. Nagy and R.D. Braatz. Open-loop and closed-loop robust optimal control of batch processes using distributional and worst-case analysis. Journal of Process Control, 14:411-422, 2004.

[16] L. F. Oliveira and P. L. D. Peres. Parameter-dependent LMIs in robust analysis: characterization of homogeneous polynomially parameterdependent solutions via LMI relaxations. IEEE Transactions on Automatic Control, 52(7):13341340, 2007.

[17] K. Zhou, J.C. Doyle, and K. Glover. Robust and optimal control. Prentice Hall, Englewood Cliffs, NJ, 1996. 